\title{
Gynaecological Pelvic Masses: A Clinical Challenge - Radiological Evaluation using Ultrasound and CT with Pathological Correlation
}

\author{
Himani Sharma ${ }^{1}$, Manish Kumar ${ }^{2}$, Atul Pandey ${ }^{3}$ Lalit Kumar ${ }^{4}$, Nishi ${ }^{5}$ \\ ${ }^{1}$ Junior Resident, Department of Radiodiagnosis, Rohilkhand Medical College, Bareilly, ${ }^{2}$ Assistant professor, Department of \\ Radiodiagnosis, Rohilkhand Medical College, Bareilly, ${ }^{3}$ Junior Resident, Department of Radiodiagnosis, Rohilkhand Medical \\ College, Bareilly. ${ }^{4}$ Professor and HOD, Department of Radiodiagnosis, Rohilkhand Medical College, Bareilly, ${ }^{5}$ Consultant \\ oncopathologist, Mahavir Cancer Sansthan, Patna, India
}

Corresponding author: Dr. Manish Kumar, Assistant Professor, Department of Radiodiagnosis, Rohilkhand Medical College, India

DOI: http://dx.doi.org/10.21276/ijcmsr.2019.4.2.34

How to cite this article: Himani Sharma, Manish Kumar, Atul Pandey, Lalit Kumar, Nishi. Gynaecological Pelvic masses: a clinical challenge - radiological evaluation using ultrasound and CT with pathological correlation. International Journal of Contemporary Medicine Surgery and Radiology. 2019;4(2):B155-B160.

\section{A B S T R A C T}

Introduction: The evaluation of the female pelvis is a radiological challenge due to the large consortium of differential diagnosis in terms of the origin of masses it houses. In the general population, the prevalence of adnexal lesions is $0.17 \%-$ $5.9 \%$ in asymptomatic women and $7.1 \%-12 \%$ in symptomatic women. The radiological modalities used in evaluation are Ultrasonography,Computed Tomography and MRI. This study strives to demonstrate the role of USG and CT together in detecting the gynecological pelvic lesions,characterize them,evaluate the findings which can differentiate malignant and benign lesions and assess their diagnostic efficacy.

Material and methods: This was a prospective study done in the Department of Radiology, RMCH,Bareilly, from November 2016-2018. All the patients of suspected pelvic mass in the age group 15-45 years in stipulated period fulfilling the inclusion criteria were enrolled for study.

Results: Ovarian masses were most frequent (66\%), followed by uterine (22\%) and adnexal masses accounted for $12 \%$ of the cases. $38 \%$ of the ovarian masses were cystic, $36 \%$ were solid and rest $26 \%$ were mixed solid-cystic lesions. In uterine lesions, leiomyoma was found to be the commonest (45.5\%).

Conclusion: Of all pelvic gynecological masses,ovarian masses were the commonest.Transvaginal sonography and transabdominal sonography were very efficacious in diagnosis of gynecological masses and were diagnostic in $93.1 \%$ and $88 \%$ cases respectively. Computed tomography was diagnostic in $78 \%$ of cases.

Keywords: Gynaecological Pelvic Masses, Radiological Evaluation, Ultrasound and CT, Pathological Correlation

\section{INTRODUCTION}

The evaluation of the female pelvis is a radiological challenge due to the varied organs and the organ system it houses. Despite being less capacious than the larger abdominal cavity, the female pelvis has a larger consortium of differential diagnosis in terms of the masses it presents. The masses arising in the female pelvis can arise from the gastrointestinal tract, reproductive system or from the different mesodermal derivatives.

Gynecologic pelvic masses are an important group of diseases which affect women of all age groups, predominantly young women. In the general population, the prevalence of adnexal lesions is $0.17 \%-5.9 \%$ in asymptomatic women and $7.1 \%$ $12 \%$ in symptomatic women. ${ }^{1}$

Patients with gynecologic mass are evaluated with a combination of clinical and diagnostic imaging methods. Ultrasonography, Computed Tomography and Magnetic
Resonance Imaging have a role in detection and characterization of gynecologic masses. They supplement clinical staging, help in preoperative planning for surgery, and assess patients for tumor recurrence.

Pelvic sonography has important role in examining a pelvic mass that may have been suspected on clinical examination. It is particularly used in patients in whom a poorly defined pelvic mass is found on examination. ${ }^{2}$ USG (both transabdominal \& transvaginal) has a primary role in detecting and characterizing uterine, endometrial and adnexal pathology.Currently, the main role of US in gynecological oncology includes evaluation of a suspected pelvic mass, evaluation of causes of uterine enlargement, identification of endometrial abnormalities in a patient with postmenopausal bleeding and characterization of ovarian masses. In addition, US has become invaluable in guiding a wide selection of invasive procedures such as transabdominal and transvaginal 
guidance of fluid or tissue sampling, transvaginal-guided drain placement and guidance for placement of brachytherapy devices for cervical and endometrial malignancies.

CT is widely used for pelvic imaging. It provides a systematic and quick overview, and coverage of abdomen is included in the same session. This makes CT well suited for tagging pelvic cancers and for imaging gynecologic and non-gynecologic diseases presenting with acute abdominal pain. CT scan is used for tumor delineation, characterization and increasing the conspicuity of peritoneal implants. Now, with the unique ability of CT to image the pelvic area of involvement, axial scanning, sagittal reconstruction and 3D reconstruction imaging of the lesions has been revolutionized. It is crucial for the characterization of adnexal masses, particularly in differentiating fluid-filled bowel loops, cystic adnexal tumors, and tubo-ovarian abscesses. It also improves the identification of hydrosalpinx and pyosalpinx. Intravenous contrast opacification is pivotal for assessing adnexal lesions, staging uterine and ovarian cancer, and assessing vascular or inflammatory diseases. ${ }^{3}$

\section{MATERIAL AND METHODS}

Prospective study was done in the Department of Radiodiagnosis, Rohilkhand Medical College and Hospital, Bareilly from November 2016 to November 2018. All cases referred to the Department of Radiodiagnosis, Rohilkhand Medical College and Hospital, Bareilly for Ultrasonography and CT scanning from Department of Obstetrics and Gynecology with clinically suspected pelvic masses were included in study. All the patients of suspected pelvic mass in the age group 15-45 years in stipulated period fulfilling the inclusion criteria were enrolled for study.

\section{Inclusion criteria}

1. All patients in the age group of $15-45$ years presenting with clinically suspected masses.

2. Incidentally detected pelvic masses in patients in whom USG was done for some other indication.

\section{Exclusion criteria}

1. Patients $<15$ or $>45$ years of age.

2. Patients having pelvic masses who have been treated either medically or surgically.

Methods of Collection of Data: After history taking, the pelvic masses were prospectively studied by both USG and CT scan.

i. The cases selected for the study were subjected to detailed history and relevant clinical examination.

ii. The patients suspected clinically to have pelvic mass were prospectively studied by both USG and CT scan.

iii. USG was done on GE LOGIQ V5 / PHILIPS ClearVue 350. All the patients were scanned with a curvilinear probe in supine position with full bladder. TVS was done in 45 patients with an endovaginal probe.

iv. Subsequent to ultrasonographic assessment, contrast enhanced computed tomography of abdomen and/or pelvis was done on GE Brightspeed 16 Slice MDCT scanner. Patients were instructed to come after overnight fast $1 \mathrm{hr}$ before the schedule examination to achieve successful bowel opacification. 3\% Gastrograffin (Trazogastro) was used as an oral contrast agent. The patients were required to drink $500-2000 \mathrm{ml}$ of $3 \%$ trazogastro solution of before the examination at steady rate of 1 cup every 10 minutes. Immediately before commencing the scan and before the patient was laid on the scan table, final cup of oral contrast agent was given in order to obtain opacification of the stomach, duodenum and proximal jejunal loops. Images were then obtained using helical data acquisition with 10 $\mathrm{mm}$ section thickness and pitch of 1.75:1 after giving IV contrast agent (60-80 $\mathrm{ml}$ of omnipaque). Thin slices $3 \mathrm{~mm}$ or $5 \mathrm{~mm}$ were also obtained wherever needed. Appropriate reconstructions were done wherever required.

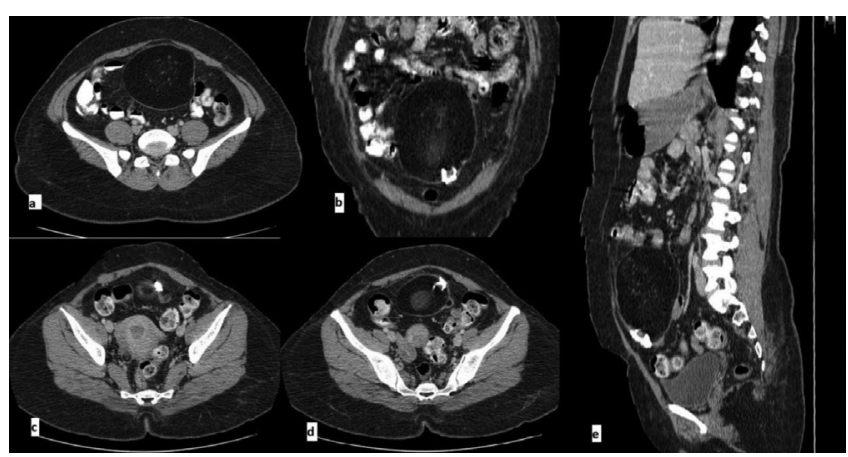

Figure-1: Benign Teratoma. Axial CECT images (a,c,d) showing a thin walled fat-attenuating lesion in the pelvis. Some soft tissue component was seen centrally which did not show significant enhancement. Coronal (b) and Sagittal (e) CT images show a hyperdense tooth-like structure eccentrically attached to wall.

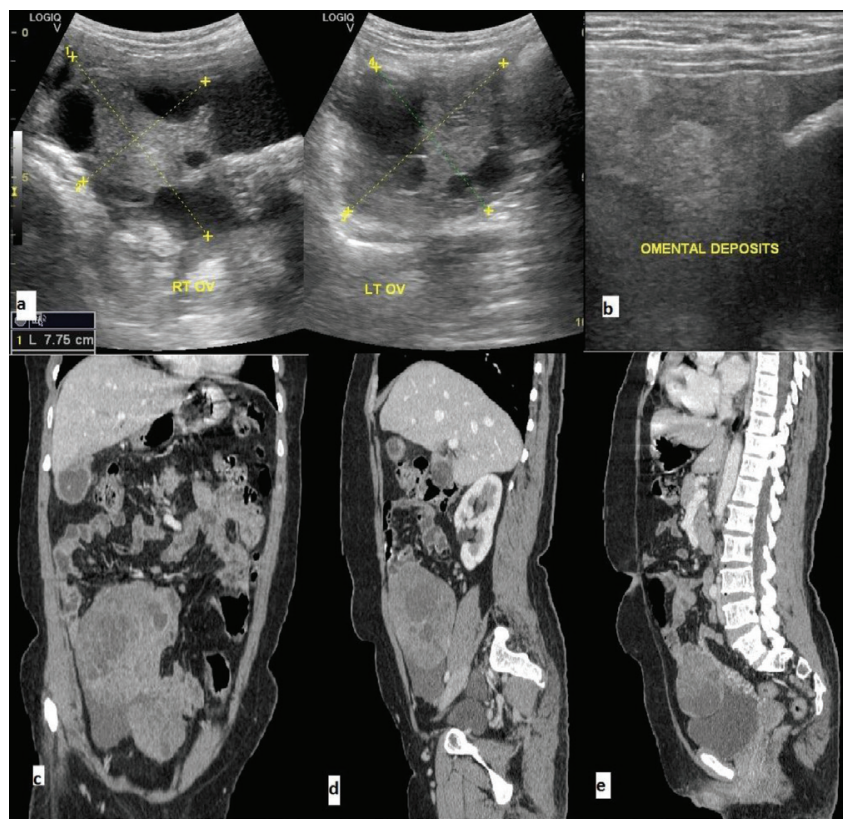

Figure-2:Krukenberg tumor.TAS image (a) showing bilateral ovarian solid-cystic masses. (b) Omental thickening was seen which turned out to be omental deposits histopathologically. Coronal (c) and sagittal (d,e) CECT images showing solidcystic masses involving both ovaries. The primary lesion was from adenocarcinoma GB. 


\section{RESULTS}

\section{Age distribution}

50 patients in the age group of 15-45 years were evaluated (Table 1). Maximum number of Ovarian masses ( $\mathrm{n}=20,40 \%)$ were seen in age group of 25-34 years. The uterine masses did not show any significant age predilection. However, all the

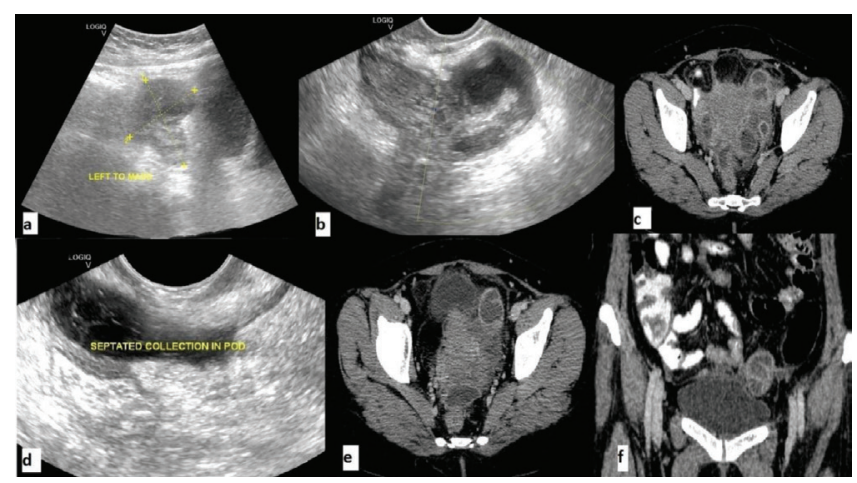

Figure-3: Left tubal abscess with pelvic inflammatory disease (a) TAS image showing a left-sided tubo-ovarian mass. (b,d) TVS image showing the thickened dilated tube having internal echoes within. Left ovary was seen separate (Not shown). A septated collection was seen in POD. (c,e) Axial and (f) coronal CECT images showing dilated left tube with enhancing collection in POD. malignant uterine masses were seen in advanced age group (35- 45 years).

\section{Organ of origin}

Ovarian masses were most frequent (66\%), followed by uterine $(22 \%)$ and adnexal masses accounted for $12 \%$ of the cases.

\section{Size distribution:}

15 out of 50 patients $(30 \%)$ had masses $<5 \mathrm{~cm}$ in size.Majority of the malignant ovarian masses (64.3\%) were between 5-10 $\mathrm{cm}$. All the malignant uterine masses were $>5 \mathrm{~cm}$.

\section{USG and CT features of masses}

The criteria used for assessment of masses were their location, extent, size, margins, septal thickness and presence of solid areas by both transvaginal and transabdominal ultrasound. $38 \%$ of the masses were cystic, $36 \%$ were solid and rest $26 \%$ were mixed solid-cystic lesions. In the ovarian lesions, septae were seen in 5 out of 33 cases (15.1\%). In most of the cases the septae were $<3 \mathrm{~mm}$ in thickness (4 out of $33,12.1 \%$ ). Solid areas were noted in 18 of 33 cases of ovarian lesions (54.5\%) (Table 2).

\section{Comparative accuracy of TAS, TVS and CT}

In 4 cases of ovarian masses, TVS was not done as the patients were unmarried.In 1 case of uterine mass, TVS was not done.Total number of cases in which TVS was done

\begin{tabular}{|l|l|c|c|c|c|c|c|}
\hline \multirow{2}{*}{ Age Group (in years) } & \multirow{2}{*}{ No. of Patients } & \multicolumn{2}{|c|}{ Ovarian masses } & \multicolumn{2}{c|}{ Uterine masses } & \multicolumn{2}{c|}{ Non Ovarian adnexal masses } \\
\cline { 3 - 8 } & & No. & $\%$ & No. & $\%$ & No. & $\%$ \\
\hline $15-24$ & 11 & 4 & $8 \%$ & 4 & $8 \%$ & 3 & $6 \%$ \\
\hline $25-34$ & 25 & 20 & $40 \%$ & 4 & $8 \%$ & 1 & $2 \%$ \\
\hline $35-45$ & 14 & 9 & $18 \%$ & 3 & $6 \%$ & 2 & $4 \%$ \\
\hline Total & 50 & 33 & $66 \%$ & 11 & $22 \%$ & 6 & $12 \%$ \\
\hline \multicolumn{7}{|c|}{ Table-1: Age Distribution of Pelvic Masses. } \\
\hline
\end{tabular}

\begin{tabular}{|c|c|c|c|c|}
\hline Characteristics of mass & Number of Patients (USG) & Percentage (USG) & Number of Patients (CT) & Percentage (CT) \\
\hline \multicolumn{5}{|l|}{ Extent of the mass } \\
\hline Limited to pelvis & 43 & $86 \%$ & 43 & $86 \%$ \\
\hline Extending to the abdomen & 7 & $14 \%$ & 7 & $14 \%$ \\
\hline \multicolumn{5}{|l|}{ Margins: } \\
\hline Well defined/regular & 36 & $72 \%$ & 36 & $72 \%$ \\
\hline III defined/irregular & 14 & $28 \%$ & 14 & $28 \%$ \\
\hline \multicolumn{5}{|l|}{ Consistency: } \\
\hline Cystic & 19 & $38 \%$ & 18 & $36 \%$ \\
\hline Solid & 18 & $36 \%$ & 14 & $28 \%$ \\
\hline Mixed & 13 & $26 \%$ & 18 & $36 \%$ \\
\hline Calcification & 7 & $14 \%$ & 7 & $14 \%$ \\
\hline Necrosis & 14 & $28 \%$ & 22 & $44 \%$ \\
\hline \multicolumn{5}{|l|}{ Septae (For ovarian lesions) } \\
\hline No Septa or Papillae & 2 & $6 \%$ & 2 & $6 \%$ \\
\hline Thin septae or papillae $<3 \mathrm{~mm}$ & 4 & $12.1 \%$ & 4 & $12 \%$ \\
\hline septa $>3 \mathrm{~mm}$ & 1 & $3 \%$ & 1 & $3 \%$ \\
\hline \multicolumn{5}{|l|}{ Solid Areas (For ovarian lesions) } \\
\hline Solid area $<1 / 3$ rd of mass & 2 & $6 \%$ & 2 & $6 \%$ \\
\hline Solid area $1 / 3-1 / 2$ of the mass & 10 & $33.3 \%$ & 10 & $30.3 \%$ \\
\hline Solid area $>1 / 2$ of the mass & 6 & $18 \%$ & 6 & $18 \%$ \\
\hline
\end{tabular}




\begin{tabular}{|c|c|c|c|c|c|c|c|c|c|c|c|c|}
\hline \multirow[t]{2}{*}{ Mass } & \multicolumn{3}{|c|}{ Clinical } & \multicolumn{3}{|c|}{ TAS } & \multicolumn{3}{|c|}{ Transvaginal Sonography } & \multicolumn{3}{|c|}{ CT scan } \\
\hline & Total & No. & $\%$ & Total & No. & $\%$ & Total & No. & $\%$ & Total & No. & $\%$ \\
\hline Benign Ovarian & 19 & 9 & 47.3 & 19 & 18 & 94.7 & 16 & 15 & 93.7 & 19 & 17 & 89.4 \\
\hline Malignant ovarian & 14 & 6 & 42.8 & 14 & 12 & 85.7 & 13 & 12 & 92.3 & 14 & 11 & 78.5 \\
\hline Benign uterine & 8 & 5 & 62.5 & 8 & 7 & 87.5 & 7 & 7 & 100 & 8 & 5 & 62.5 \\
\hline Malignant uterine & 3 & 2 & 66.6 & 3 & 3 & 100 & 3 & 3 & 100 & 3 & 2 & 66.6 \\
\hline Adnexal masses & 6 & 2 & 33.3 & 6 & 4 & 66.6 & 6 & 5 & 83.3 & 6 & 4 & 66.6 \\
\hline Total & 50 & 24 & 48 & 50 & 44 & 88 & 45 & 42 & 93.3 & 50 & 39 & 78 \\
\hline
\end{tabular}

Table-3: Diagnostic accuracy of clinical examination, transabdominal sonography, transvaginal sonography and computed tomography.

was 45.Transabdominal ultrasound was able to detect origin in 44 of 50 cases, while 6 cases remained indeterminate. Transvaginal sonography had an overall accuracy of $88.8 \%$ in evaluation of the organ of origin. In 5 of the patients, TVS could not be done as the patients were unmarried.Computed Tomography had an overall accuracy of $78 \%$ while it remained indeterminate in the rest 22\% (Table 3).

\section{Extra pelvic abnormalities:}

CT and ultrasound had equal sensitivity in detecting liver metastases. CT was better in delineation of peritoneal metastases. CT was also found to be superior in detection of lymphadenopathy and in the assessment of local infiltration.

\section{Histological types of lesions}

a) Uterine

- Leiomyoma- $45.4 \%$

- Congenital uterine lesions- $18.2 \%$

- $\quad$ Endometrial polyp- 9.1\%

- Uterine malignancies- $27.3 \%$

b) Ovarian

8 out of 33 ovarian masses were non-neoplastic benign lesions, of which hemorrhagic cysts were most common. In the neoplastic benign lesions, 3 were serous cystadenomas, benign teratoma and mucinous cystadenoma accounted for 4 each, whereas 2 lesions turned out to be fibromas.Serous and mucinous tumors had equal incidence.

c) Adnexal

Two of the tubal abscesses were observed with serial ultrasound and they were seen to resolve. In the remaining 2 cases, histopathology was obtained.

\section{DISCUSSION}

The present series consist of 50 female patients referred from the department of obstetrics and gynecology of Rohilkhand Medical College, Bareilly. Etiologically, pelvic masses can be broadly divided into gynecologic and non-gynecologic group. Our study included only the gynecological cases. Ovarian masses formed the largest subgroup accounting for $66 \%$ of cases (33 of the 50 pelvic masses). Uterine (22\%) and non - ovarian adnexal (12\%) were the next most common masses observed.

\section{Radiological spectrum}

The radiological investigations that were performed included transabdominal sonography, transvaginal sonography and computed tomography. The gynaecologic pelvic masses are characterized according to their location, extent, size, margins, internal architecture and adjacent infiltration.
$38 \%$ of all observed masses were cystic, $36 \%$ solid and $26 \%$ were mixed on sonography. Almost similar sonography spectrum was observed in Lawson et al in $1977 .{ }^{4}$

TVS is the best imaging modality for differentiation of tubal from ovarian pathologies. TVS clearly delineated the anatomy of the dilated and tortuous fallopian tube, which was diagnosed as tubo-ovarian abscess on transabdominal sonography. Lande and associated reported similar findings. ${ }^{5}$ The major limitation that was observed on transvaginal sonography was the inability of global demonstration of the pathological process and failure to define the anatomical relationships of a large mass. These findings are similar to the observation made by Mendelson et al in $1988 .^{6}$ This is in accordance to the previous reports, which have been reported highly accuracy of transvaginal sonography over transabdominal sonography in evaluation of gynecological masses. ${ }^{7,8}$

Computed tomography classified ovarian masses as malignant based on the criteria of septal thickness, solid areas and wall irregularities (Table-2). Thus, computed tomography detected feature of malignancy in $78.5 \%$ (11 out of 14) of ovarian malignancies. It is slightly less than the $94 \%$ detection rate obtained by Fakuda et al in $1986 .{ }^{9}$

Uterine masses mainly appeared solid on computed tomography. Endometrial adenocarcinomas demonstrated non-enhancing hypodense areas with surrounding infiltration.

CT was superior to TAS in malignant masses, in defining the local spread. Local infiltration was seen in $26 \%$ of cases with CT compared to $16 \%$ of cases with TAS. Osaza observed a higher proficiency of $\mathrm{CT}$ in predicting adhesions around the mass. ${ }^{10}$

\section{Specific masses and their characteristics \\ Ovarian masses}

Non neoplastic ovarian lesions constituted 8 out of 33 cases, 5 out of which were hemorrhagic cysts and 3 were endometriotic cysts. On ultrasound, hemorrhagic cysts were seen as cystic lesions with dependent debris, cystic lesions with a mobile echogenic clot or cystic lesions having honeycomb appearance. On CT, the hemorrhagic cysts demonstrated minimal to mild wall enhancement without any enhancing solid component. Endometriotic cysts were seen as well defined cystic lesions with homogeneous low to medium level internal echoes and posterior acoustic enhancement on ultrasound. On CT endometriomas were seen as cystic lesions with a well-defined wall and internal hyperdense contents. 
Epithelial tumors were seen in $42.2 \%$ (14 out of 33) as against a reported incidence of $90 \%{ }^{11}$

All benign mucinous cystadenomas demonstrated septae or solid areas $<3 \mathrm{~mm}$ size. All malignant mucinous tumors demonstrated solid areas involving atleast one-third to onehalf of the mass, similar to findings of Fakuda et al in $1986 .{ }^{9}$ $83.3 \%$ of papillary serous carcinoma demonstrated solid areas occupying $>1 / 2$ of the mass. Calcification was seen in $14 \%$ of ovarian tumors. Although peritoneal metastases are more common with serous carcinoma, we observed equal incidence in mucinous tumors. This could be attributed to larger number of mucinous cystadenocarcinomas included in our study.

2 cases of fibroma ovary were evaluated. 1 of them could not be characterized on sonography due to marked attenuation of the beam. CT demonstrated solid appearance with calcification and few necrotic areas.

Germ cell tumors constituted $21.1 \%$ of ovarian masses. 4 cases of benign teratomas were studied measuring between 5-10 cm. 1 case of malignant teratoma was studied which was $>15 \mathrm{~cm}$ in size. The 2 cases of germ cell malignancies were also seen in younger age group of less than 25 yrs. Majority of benign cystic teratomas demonstrated typical sonographic pattern of calcification, mural nodule and echogenic areas with posterior acoustic shadowing on USG. These were fat or high density fluid on CT, often containing calcification/ hyperdense elements(Fig 1). ${ }^{12} \mathrm{CT}$ detected fat in all cases similar to the reported incidence of $93 \%$ by Buy et al in 1989. ${ }^{13}$

The incidence of metastatic tumor to ovaries is reported as $3-8 \%$ similar to the observed incidence of $6 \%$ in our study. The common primaries reported to metastasize to ovaries are GIT and breast malignancies. ${ }^{14}$ Metastatic GB carcinoma showed typical fairly homogenous bilateral solid ovarian masses with few necrotic areas in our study (Fig 2).

The overall diagnostic accuracy by Transabdominal sonography in ovarian mass was $90.9 \%$ which has significant improvement over $68 \%$ seen in Benacerraf series. The probable cause of difference could be a larger variety of ovarian lesions studied by us compared to only epithelial tumors evaluated by them.

\section{Uterine masses}

11 uterine masses were studied. 5 cases of leiomyoma uterus were studied by us. Patients with fundal leiomyoma presented with menorrhagia and were accurately diagnosed by all imaging modalities. We observed similar CT appearance in leiomyomas as described by Gross in $1987 . .^{15} 1$ case of endometrial polyp was studied, which was seen extending upto the cervical canal through a stalk attached to the fundal region. Doppler demonstrated vascularity in the stalk.

Our results in benign uterine tumors were similar with both the imaging modalities, thus sonographic study maybe sufficient in diagnosis of such masses considering the radiation hazard and high cost of $\mathrm{CT}$ as it adds no further useful information in most of the cases.

The reported incidence of developmental uterine anomalies is $0.1-0.5 \%$ of the population but usually anomalies were detected as pelvic masses when associated with hematometra or hematocolpos.

3 cases of malignancy of uterus were included in the study, out of which 1 was a case of cervical carcinoma. All the cases were diagnosed on both USG and CT but accurate staging was possible only in CT. CT is known to play a limited role in early endometrial carcinoma, however its role in staging of bulky and advanced malignancies as seen in our study is well established. ${ }^{16}$

\section{Non ovarian adnexal masses}

We studied 6 cases of adnexal lesions; 4 of them were tubal abscesses which were best demonstrated with TVS. CT demonstrated the pathological process in cases of multiple pelvic abscesses accurately, clearly showing the loss of fat planes and contrast enhancing inflammatory tissue in addition to cystic areas (Fig 3).

We diagnosed endometriosis with confidence based on the identification of uterine hypodensities and adnexal cysts on $\mathrm{CT}$ andTVS. Although the sonographic patterns were similar to those described by Sandler et al in 1978 and Birnholtz et al in 1983, sonographic features of Endometrioma overlapped with other cystic masses. ${ }^{16}$ Identification of irregular hypoechoic areas in myometrium suggesting adenomyosis helped in achieving the correct diagnosis.

\section{CONCLUSION}

Of all pelvic gynecological masses, ovarian masses were the commonest variety seen. It was followed in incidence by uterine masses and adnexal masses had the least incidence. Transabdominal sonography was the best imaging modality for the initial evaluation of pelvic masses being inexpensive, safe and easily available. Transvaginal sonography was most useful in the detection of organ of origin and in morphological characterization of small gynecological masses. The role of computed tomography was maximum in characterization of malignant and indeterminate masses. Computed tomography was highly efficacious for studying pelvic masses and was diagnostic in 78\% of cases. TAS and TVS were very efficacious in diagnosis of gynecological masses and were diagnostic in $93.1 \%$ and $88 \%$ cases respectively. Imaging modalities contributed significantly in diagnosing and characterization pelvic masses. Therefore, combining the transabdominal, transvaginal and computed Tomography, conclusive diagnosis was achieved in almost all cases.

\section{REFERENCES}

1. Rathore OP. Radiopathological correlation of adnexal lesions: Our experience. JMSCR. 2017; 05(07): 2487624886.

2. Kurjak A, Prka M. Ovarian Sonography. Donald School Of Ultrasound in Obstetrics and Gynaecology. 2004; $1^{\text {st }}$ ed: 610

3. Fortner R, Kinkel K Female pelvis. Computed tomography and Magnetic Resonance Imaging of the whole body. 2002; 1 (4 $4^{\text {th }}$ ed): 2075-2076

4. Seshul B.M., Coulam C.M. Pseudomyxoma peritonei: Computed tomography and sonography, AJR 1981;136: 803-806.

5. Fakuda T., Ikeuchi M. and Hashimoto H. et al . Computed Tomography of Ovarian Masses. J Comput 
Assist Tomogr 1986;10(6): 990-996.

6. Azoury B., Woodruff J.D. Primary Ovarian Sarcoma. Report of 43 cases Emil Novak Ovarian Tumor Registry. Obstet \& Gynecol 1971;37(3):920-922.

7. Bierman S.M., Reuter K.L., Hunter R.E. Meigs syndrome and ovarian fibroma: CT findings. J Computed Tomography 1990;14 (5): 833-834.

8. Roche et al. Radiological appearances of Gynaecological emergencies. Insights Imaging. 2012; 3(1):265-275.

9. Medelson E.B., Bohm-Velez M., Joseph N. et al . Gynecologic Imaging: Comparison of Transabdominal sonography and Transvaginal sonography Radiology 1988;166(5): 321-324.

10. Athey P.A., Cooper N.B. Sonographic Features of Paraovarian Cysts. AJR 1985;144(6):83-86.

11. Dharita et al. Doppler Ultrasound: A Good and Reliable Predictor of Ovarian Malignancy. The Journal of Obstetrics and Gynecology of India. 2013;63(3):186189.

12. Walsh JW, Rosenfield AT. Prospective comparison of ultrasound and computed tomography in the evaluation of gynecologic pelvic masses. Am J Roentgenol 1978;131(5):955-960.

13. Ong S., Duffy T., Murphy J. Transabdominal ultrasound and its correlation with clinical findings in gynecology. Ir J Med Sci. 1996; 165(4): 168-270.

14. Abu Rustam N.R, Aghajanian C.A, Venkataraman E.S et al. Metastatic Breast Carcinoma to the Abdomen and Pelvis. Gynecol Oncol 1997;66(1): 41-44.

15. Mitchell D.G., Mintz M.C. and Spritzer C.E. et al. Adnexal masses: MR Imaging Observation at $1.5 \mathrm{~T}$, with US and CT correlation. Radiology 1987;162(5): 319-324.

16. Sandler M.A., Silver T.M. and Karo J.J. gray scale ultrasonic features of ovarian teratoma. Radiology 1979; 131(3): 705-709.

Source of Support: Nil; Conflict of Interest: None

Submitted: 20-04-2019; Accepted: 15-05-2019; Published online: 30-06-2019 